\title{
ARQUITECTURA SISMO-RESISTENTE. UN NUEVO ENFOQUE PARA LA SOLUCION INTEGRAL DEL PROBLEMA SISMICO
}

\author{
(SEISMO-RESISTANT ARCHITECTURE)
}

Hugo Giuliani, Ingeniero*

Adriana Citrinovitz, Silvia Aladro y Hemilce Benavidez, Arquitectos ${ }^{\star}$

\section{RESUMEN}

El presente trabajo, sólo pretende la formulación en términos generales de las razones, fundamentos. objetivos, campos de acción, principios básicos y pautas de compatibilización del Diseño Estructural con el Diseño Arquitectónico que nos han permitido plantear una Arquitectura Sismo-Resistente y que permitirá en el futuro su profundización y el desarrollo científico de la misma.

\section{SUMMARY}

This work only pretend to state in general terms the reasons, foundations, objectives, action fields, basical principles and compatibilization rules of the Structural Design with the Architectural Design that have allowed us to draw an approach on Seismo-Resistant Architecture and that in the future will allow its study and scientific development.

\section{INTRODUCCION}

Hasta hace algunos años, la solución a los problemas derivados de la excitación sísmica se limitaba, fundamentalmente, al análisis estructural. Por otra parte el advenimiento de las computadoras electrónicas hizo posible un extraordinario avance de la Ingeniería Sismoresistente. Así se lograron sofisticadas metodologías para el análisis dinámico de las más diversas estructuras. Sin embargo, los últimos terremotos dejaron experiencias que nos permiten reconocer que este avance debe ir acompañado, también, con experiencias de laboratorio $y$, en especial, no olvidar las experiencias y enseñanzas que nos deja cada terremoto.

(*) Profesor de la Facultad de Ingeniería y Arquitectura e Investigador del Instituto de Investigaciones Antisísmicas "Ing. Aldo Bruschi» - Universidad Nacional de San Juan, República Argentina.

(**) Becarias de Perfeccionamiento del Consejo Nacional de Investigaciones Científicas y Técnicas (CONICET) en el Instituto de Investigaciones Antisísmicas «Ing. Aldo Bruschis - Universidad Nacional de San Juan, República Argentina.
En síntesis, los últimos terremotos que afectaron ciudades importantes con edificios construidos satisfaciendo prescripciones de normas sismoresistentes vigentes han demostrado, además de que dichas normas han resultado insuficientes en muchos casos, que el sólo análisis estructural no es suficiente para asegurar la estabilidad y objetivos de una construcción en zonas sísmicas. Ha quedado en evidencia la necesidad del Diseño Estructural Sismo-resistente y su rol en el verdadero comportameinto de las construcciones frente al sismo.

En tal sentido los especialistas de Ingeniería Sismo-resistente han elaborado una serie de recomendaciones para ser tenidas en cuenta en el Diseño Estructural y también en el Diseño Arquitectónico.

Sin embargo, el tema así enfocado se reduce a limitadas recomendaciones tendentes a evitar determinadas situaciones que ponen en peligro el correcto comportamiento sismo-resistente de los edificios, pero sin considerar el problema en forma global e integral. Es decir, un enfoque totalizador de la respuesta de las construcciones a 
las acciones sísmicas, ya que de esta respuesta no sólo es responsable la estructura resistente, sino todos los elementos que materializan el edificio y la ciudad.

Esto nos indica que la solución sismo-resistente comienza a gestarse en la etapa de análisis del proceso proyectual y que no se limita al análisis y diseño estructural.

El objetivo es que el ARQUITECTO como responsable directo en el diseño de los espacios urbanos y edificios, asuma la responsabilidad de la sismo-resistencia en la etapa de proyecto. Para esto es necesario crear conciencia entre los arquitectos de que sólo a través del trabajo interdisciplinario de Ingenieros y Arquitectos podrán proponerse soluciones que aseguren un adecuado Diseño Arquitectónico Sismo-resistente.

Sin duda, no resulta fácil asumir este enfoque en plenitud, ya que significa modificar la actitud mental del Arquitecto que considera al tema de exclusiva responsabilidad del Ingeniero estructural sismo-resistente.

Son varios los motivos que lo llevan a mantener esta actitud, entre ellos:

- Desconocimiento de los conceptos básicos de Sismología y de Ingeniería Sismo-resistente.

- Falta de claridad y precisión respecto del tema: Enseñanza de las Estructuras a los alumnos de Arquitectura.

- La notable diferencia existente en la formación científica de los especialistas de la Ingeniería sismo-resistente y de los Arquitectos.

Desde 1982, el Instituto de Investigaciones Antisísmicas «Ing. Aldo Bruschi», Facultad de Ingeniería, Universidad Nacional de San Juan, viene desarrollando una Arquitectura Sismo-resistente sobre la base de dicho enfoque. El equipo interdisciplinario está constituido precisamente por los autores de este trabajo, que expone brevemente los resultados, avances y conclusiones obtenidas.

Los primeros trabajos fueron presentados en las "4. a Jornadas Chilenas de Sismología e Ingeniería Antisísmica». Allí el tema despertó interés entre los Arquitectos que vislumbraron con certeza un camino que les permitirá asumir su responsabilidad en el problema sísmico.

Posteriormente se realizaron las Primeras Jornadas Argentinas de Arquitectura Sismo-resistente. Estas Jornadas fueronorganizadas por el Instituto Regional de Planeamiento y Hábitat de la Facultad de Arquitectura de la Universidad Nacional de San Juan. En esta oportunidad nuestros trabajos permitieron a los participantes apreciar con claridad los fundamentos y objetivos de este enfoque.

Por otra parte el tema así planteado y como consecuencia de la divulgación lograda, seguramente ha llevado a organizar para el año 1987 el Primer Congreso Internacional de Arquitectura en Zona Sísmica a realizarse en Mendoza, Argentina.

\section{ARQUITECTURA SISMO-RESISTENTE}

\section{Emergencia sísmica}

La denominación elegida se presta a ciertas polémicas pero, sin embargo, preferimos seguir con esta denominación por cuanto se trata de poner en evidencia un calificativo, a la Arquitectura, de gran importancia en Zonas de Alto Riesgo Sísmico. Dicha importancia reside en el hecho de lograr su invulnerabilidad frente a los terremotos. De su sismo-resistencia depende la propia existencia no sólo del edificio y de la ciudad, sino la de sus habitantes y bienes económicos.

El desarrollo científico de una Arquitectura Sismorresistente implica desarrollar tres líneas de estudio:

a) Evaluar la vulnerabilidad sísmica de las zonas urbanizadas.

b) Elaboración de propuestas para la mitigación 'de los efectos destructivos de los terremotos que afectarán a las zonas urbanizadas.

c) Planificación de la Emergencia Sísmica.

\section{Planificación urbana sismo-resistente}

a) Incluir al sismo como condicionante del comportamiento urbano.

b) Desarrollar pautas generales de diseño para la prevención y mitigación de los efectos de sismos destructivos.

\section{Arquitectura sismo-resistente}

a) Establecimiento de los principios básicos generadores de la arquitectura sismo-resistente.

b) Planteo y perfeccionamiento de las interrelaciones entre el diseño estructural y el diseño arquitectónico frente al condicionante sísmico.

c) Particularidades de la arquitectura sismoresistente en relación a: sistemas constructivos tradicionales e industrializados, autoconstrucción, etcétera. 


\section{PARTICULARIDADES DEL ANALISIS Y DISEÑO SISMO-RESISTENTE}

En cuanto al diseño estructural sismo-resistente presenta particularidades, como por ejemplo:

- Las fuerzas sísmicas son proporcionales al peso de la construcción, por consiguiente, es buena práctica reducir el peso de las construcciones, en la medida que sea posible.

- Otra importante característica que la diferencia del diseño estructural para cargas verticales, se refiere al hecho de que, si sólo hay traslación, cada elemento resistente absorbe una fuerza sísmica horizontal proporcional a su rigidez horizontal, independientemente de su ubicación en la planta general del piso considerado. Esto facilita notablemente el diseño, permitiéndonos disponer la ubicación de los principales elementos estructurales sismoresistentes de la manera más conveniente a fin de lograr minimizar los efectos de torsión y atender requerimientos del proyecto arquitectónico.

El comportamiento del edificio durante el terremoto depende, en mayor medida que para cargas verticales, de la suma de los comportamientos de todos los componentes de las construcciones (estructurales, no-estructurales, instalaciones, etc.).

\subsection{Ductilidad}

A diferencia de los criterios de diseño estructural para cargas verticales y estáticas, el diseño sismo-resistente se funda, especialmente, en la capacidad de deformación plástica que en su propia capacidad de resistencia.

El beneficio de las deformaciones plásticas se traduce en las estructuras sismo-resistentes de dos modos:

- Disipación de energía cinética o sísmica en el trabajo de deformación.

- Redistribución de tensiones y esfuerzos permitiendo una mayor eficacia de las estructuras.

Lógicamente, las estructuras de alto grado de hiperestaticidad, han aumentado el número de articulaciones plásticas, actuando como disipadores de energía sísmica.

\subsection{Filosofía del diseño sismo-resistente}

Es importante entender cuáles son los alcances de un diseño sismo-resistente y cuáles son sus características especiales. Debemos empezar por aceptar que el propósito básico de acuerdo con los objetivos del diseño sismo-resistente ya analizados, es la ejecución de construcciones seguras a los efectos sísmicos con el fin de evitar: pérdida de vidas, el colapso de las mismas, aceptando daños menores económicamente reparables y asegurar la continuidad de funcionamiento de los servicios vitales, ante la ocurrencia del máximo terremoto probable.

De acuerdo con esto es evidente que no se pretende la preservación total de las construcciones, pero sí evitar pérdida de vidas y el colapso total de las mismas. Esto es un problema de conciliar seguridad con economía, donde deben evaluarse una serie de factores; entre ellos podemos indicar los siguientes:

a) Probabilidad de ocurrencia del terremoto destructivo y de mediana intensidad.

b) Incidencia del mayor costo de la estructura sismorresistente.

c) Costo de reparación de los daños admitidos.

d) Destino de las construcciones.

En efecto, se trata en general de que las construcciones puedan resistir sin daños los sismos de mediana intensidad y de mayor probabilidad de ocurrencia y de admitir daños económicamente reparables, para los sismos destructivos de poca probabilidad de ocurrencia. Pero es evidente que hay construcciones que, por su destino, necesitan mayor seguridad, como por ejemplo las usinas termo-nucleares, donde en ciertas partes de las mismas es inadmisible cualquier tipo de daño. También se necesita en los edificios públicos como: escuelas, hospitales y otras que proveen servicios cuya continuidad son especialmente necesarios después del terremoto.

Desde el punto de vista estructural lo que se pretende es que las construcciones permanezcan dentro del campo elástico para los sismos más frecuentes y, en cambio, incursionen dentro del campo elasto-plástico para los sismos destructivos, cuya probabilidad de ocurrencia es muy reducida.

Consecuentemente, la filosofía evidentemente del cálculo y proyecto sismo-resistente de las construcciones es diferente de las correspondientes para cargas permanentes debidas al peso propio y sobre cargas.

Por otra parte, el carácter dinámico de la excitación sísmica condiciona una respuesta no sólo de la estructura resistente sino también la de los elementos no resistentes de las construcciones. Consecuentemente los elementos no estructurales interfieren el comportamiento previsto para la estructura, pudiendo mejorarlo o deteriorarlo 
hasta el punto de ser la causa de su colapso por escalonamiento de su capacidad de resistencia sísmica.

Surge así con total claridad la naturaleza de la responsabilidad del arquitecto en zonas sísmicas de alto riesgo frente a la necesidad de un enfoque totalizador de la sismo-resistencia.

\section{PRINCIPIO BASICO}

Hemos establecido el siguiente principio fundamental para el diseño estructural, que a nuestro juicio debe cumplirse bajo riesgo de colapso de la estructura durante un sismo. Principio, que a su vez, se ha convertido en el «Principio básico de la arquitectura sismo-resistente»:

"Las estructuras sismo-resistentes deben diseñarse de tal forma que todos sus elementos resistentes puedan actuar simultáneamente.»

Desde el punto de vista del diseño estructural, esto se logra en la medida que la rigideźresitencia sea la misma en todos sus elementos y tanto en fase elástica como en la elastoplástica.

En caso contrario es fácil comprender que se producirá la rotura escalonada de los elementos o dispositivos resistentes llevando lógicamente al colapso la estructura resistente.

Corresponde también, al diseño arquitectónico sismo-resistente, compatibilizar sus diversos aspectos: funcional, formal espacial, constructivo, económico, estético, de tal manera que satisfaga las exigencias de este principio.

En síntesis la teoría, la metodología, la investigación que sirvan para desarrollar una arquitectura sismo-resistente, debe inevitablemente satisfacer este principio fundamental. Es por esto que lo formulamos como el Principio Básico de la Arquitectura Sismo-resistente.

\section{OBJETIVOS DE EFICIENCIA Y OPTIMIZACION DE LA RESPUESTA SISMO-RESISTENTE DE LOS EDIFICIOS}

Este objetivo se puede lograr en principio de dos maneras, una reduciendo las fuerzas sismicas y otra aumentando la eficiencia de la capacidad sismo-resistente de los edificios.

La reducción de las fuerzas sísmicas se puede obtener de diversas formas, a saber:

Reduciendo el peso de la construcción, lo que implica utilizar materiales livianos.

Diseño arquitectónico: También es muy importante tener en cuenta este objetivo durante el diseño arquitectónico. En efecto, se recomienda ubicar los locales destinados a archivos, piletas de natación, o equipos pesados en los niveles inferiores, siempre que ello sea factible.

Se consigue así, no sólo reducir las fuerzas sísmicas por el hecho que el coeficiente sísmico aumenta en los niveles superiores, sino también reducir, lógicamente, los momentos y corte sísmico.

Evitando la seudo resonancia: Esto implica evitar que el período fundamental del edificio coincida con el período predominante del terreno de fundación.

Por otra parte, la optimización de la capacidad sismo-resistente se puede obtener procurando formas espaciales que conduzcan a una estructura clara, simple y con centro de torsión coincidente con el centro de masa que permita a su vez optimizar el diseño estructural. Este objetivo, sin duda, constituye un claro desafío a la arquitectura que implica nuevas investigaciones y estudios para que desde el diseño arquitectónico se hagan aportes significativos a la mejor solución del problema sísmico.

\section{CAUSAS QUE PROVOCAN EL COLAPSO PARCIAL Y TOTAL DE LAS CONSTRUCCIONES}

Hay que distinguir si la construcción es sismoresistente o no.

En el primer caso si no hay error de cálculo estructural ni de construcción defectuosa debe suponerse que de acuerdo con las actuales normas sismo-resistentes no debe esperarse colapso parcial ni mucho menos total. Sin embargo, se producen, y las causas principales que lo provocan se deben a una de las dos siguientes:

Las fuerzas sísmicas son superiores a las previstas. Esto puede suceder debido a ciertas particularidades que circunstancialmente se olvidan.

Estas particularidades son función principalmente de las propiedades dinámicas del terreno de fundación y de la estructura geológica circundante y de la distancia epicentral. El primero por las frecuencias que introduce en el movimiento sísmico y el segundo porque las altas frecuencias van absorbiéndose a medida que aumenta la distancia recorrida por las ondas sísmicas.

Su prevención exige tener en cuenta todos los antecedentes sísmicos, geológicos de la región, y su compatibilización con las características dinámicas del edificio. Esto es lo que ocurrió en México. 
Escalonamiento de la capacidad sismoresistente, es decir, cuando no se ha cumplido con el principio fundamental del diseño estructural.

Esta situación es siempre atribuible a un mal diseño estructural o a la falta de compatibilización con el diseño arquitectónico. El resultado es impredecible porque no depende de la capacidad resistente que se supone ha sido prevista en un todo de acuerdo con normas vigentes, sino del grado de escalonamiento de la misma.

Esta situación también ha sido la otra causa de la mayoría de los colapsos en el terremoto de México. La suma de las causas señaladas han determinado el sorprendente desastre de dicha ciudad.

Esto pone en evidencia que el principio fundamental del diseño estructural debe satisfacerse en todos los casos. Esto a su vez requiere, como se ha dicho, una respuesta del diseño arquitectónico, convirtiéndose así en el principio básico de la arquitectura sismo-resistente.

Por supuesto que los errores de cálculo estructural o defectos constructivos, por sí solos, pueden conducir al colapso parcial y total. En estos casos se convierten en factores que disminuyen o causan el escalonamiento de la capacidad resistente.

Si las construcciones no son antisísmicas lo natural es que ocurra el colapso parcial o total de las mismas. La razón determinante es la falta de capacidad sismo-resistente y las causas que en particular determinan el colapso son el agotamiento de la resistencia sísmica que circunstancialmente puedan tener, agravado por las causas que se han señalado para el escalonamiento de dicha precaria resistencia sísmica.

\section{CAUSAS QUE PROVOCAN EL ESCALONAMIENTO DE LA SISMO-RESISTENCIA}

Relación rigidez-resistencia (causas inherentes al propio diseño estructural).

Recordemos que los elementos resistentes más rígidos absorben más corte sísmico que los más flexibles. En consecuencia deberán ser también más resistentes, es decir, que la "resistencia de todo elemento estructural debe ser congruente con su rigidez».

Esta necesidad de conciliar rigidez-resistencia plantea en la práctica del diseño estructural sismo-resistente numerosos casos de difícil solución. Los casos más conocidos son los siguientes:
Columnas de hormigón armado de igual altura de los pisos y sección transversal del mismo ancho pero con alturas diferentes: El problema surge porque, mientras la rigidez es proporcional al cubo de la altura, el momento flector es proporcional al cuadrado de la misma. Es decir:

$$
\begin{aligned}
R=\frac{12 E J}{H_{c}^{3}} & =\frac{12 E}{H_{c}^{3}} \cdot \frac{b h^{3}}{12} \\
M & =\frac{b}{k_{h}} h^{2}
\end{aligned}
$$

Así para el caso de una columna con altura, de la sección transversal, doble de otra que tiene el mismo ancho, resulta que su rigidez es ocho veces mayor que la de sección menor; en cambio su momento flector resistente es sólo cuatro veces mayor.

Frente a esta situación se recurre a incrementar la cuantía de acero. Pero lo cierto es que la rigidez también es función de la cuantía, cosa que en la práctica no se la tiene en cuenta y esto modifica la relación rigidez-resistencia, introduciendo un factor de incertidumbre que puede facilitar el escalonamiento de la sismo-resistencia.

Torsión sísmica: El hecho que el corte sísmico en una columna provocado por un momento torsor es proporcional a la distancia al centro de torsión o rigidez (C.T.) da como resultado que para columnas dimensionalmente iguales, pero ubicadas a distintas distancias del C.T., las fuerzas sísmicas sean diferentes. Esto, indudablemente, puede ser la causa fundamental del escalonamiento de la capacidad de resistencia sísmica, especialmente si no ha sido previsto el efecto torsional.

En efecto, si tenemos dos columnas iguales pero una a una distancia triple de la otra, la fuerza sísmica de la primera es también triple de la segunda; se comprende que estamos ante un problema de difícil solución en el sentido de dotar a las dos columnas de una misma relación rigidezresistencia.

Sabemos que en la práctica se recurre a modificar la cuantía de aceros o bien la forma de la sección transversal, pero estos recursos no nos aseguran la relación rigidez-resistencia necesaria.

El momento torsor resulta, en el caso de losas rígidas, cuando el centro de torsión (C.T.) no es coincidente con el centro de masas (C.M.). En estos casos los efectos de la torsión sísmica pueden determinarse pero subsiste la dificultad planteada anteriormente de mantener la relàción rigidez-resistencia para las columnas.

Hay otros casos en que el momento torsor aparece por deformabilidad de los entrepisos en casos especiales, aun cuando el C.T. coincide con 


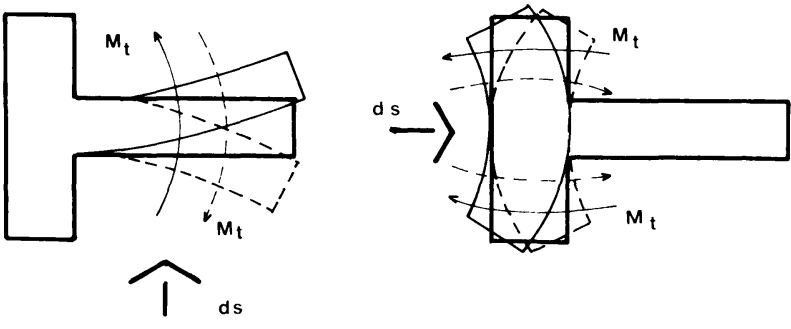

el C.M. Estos casos se dan en plantas como las indicadas en la figura.

Estas situaciones, en la práctica corriente, no se las tiene debidamente en cuenta. Sin embargo, puede ser la causa del escalonamiento de la sismo-resistencia.

Piso flexible: La existencia de un piso mucho más flexible que los restantes trae consigo el siguiente problema. Al ocurrir sismos muy fuertes el corte sísmico de los entrepisos más rígidos se verán reducidos y en cambio en el flexible se verá incrementado. Este requiere que la ductilidad y resistencia del piso flexible sea muy superior que la requerida cuando todos los pisos tienen aproximadamente la misma rigidez.

Los ejemplos de construcciones como el Hospital Olive View (California) y el bloque S-E de la Escuela ENET n. ${ }^{\circ} 2$ (San Juan), ambas con plantas bajas flexibles que fallaron al ser sometidas a los terremotos de San Fernando (1970) y San Juan (1977), respectivamente, indican la inconveniencia de estos diseños estructurales.

Nuevamente estamos ante un escalonamiento de la capacidad de resistencia sísmica: En estos casos, el determinado por la modificación de la relación rigidez-resistencia del piso flexible, por la particular ausencia de muros. De manera que la solución consiste en establecer la necesaria relación rigidez-resistencia del piso flexible y rechazar la posibilidad teórica de utilizarlo para mejorar el comportamiento sismo-resistente del conjunto del edificio.

Columna corta: Otro tema ligado al problema rigidez-resistencia es el denominado "columna corta». Aquí el corte sísmico aumenta inversamente proporcional al cubo de su altura para columnas de igual área de sección transversal.

Además, para columnas de muy poca altura esto se agrava por el hecho de que el hormigón no es apto para resistir fuertes esfuerzos tangenciales, disminuyendo notablemente su ductilidad.

Estos casos se producen por una disposición particular de las mamposterías, que reducen la altura de las columnas y consecuentemente su rigidez se incrementa mucho más. Esto hace que se concentre un notable corte sísmico, que lógicamente no puede resistir. La rotura de es- tos elementos resistentes deja indefenso al resto de los elementos resistentes, pudiendo sobrevenir el colapso total.

Las columnas cortas deben evitarse en el diseño estructural y arquitectónico de los edificios y si resultan inevitables deben dotarse de la necesaria capacidad resistente. Muchos son los casos de colapso parcial y total de construcciones debido a este efecto no tenido debidamente en cuenta durante su diseño y cálculo sismoresistente.

Vigas cortas: Cuando en un pórtico una de las vigas en un determinado piso o nivel es de una longitud notablemente menor que las restantes se produce el caso denominado viga corta. Aquí el problema surge porque la rigidez angular es inversamente proporcional a su longitud, es decir:

$$
R=\frac{4 E J}{1_{v}}
$$

La dificultad es, como en los casos anteriores, lograr la necesaria relación rigidez-resistencia.

La evidente concentración del momento flector puede provocar su rotura y facilitar, lógicamente, el escalonamiento e la capacidad de resistencia sísmica del conjunto estructural.

Elementos no estructurales: Se sabe que los elementos no estructurales, tales como muros, tabiques divisorios, instalaciones, etc., interfieren el comportamiento previsto para la estructura resistente. Esta interferencia puede ser en el sentido positivo y también negativo. Son muchos los casos en donde éste ha sido el motivo principal del escalonamiento de la capacidad de resistencia sísmica del conjunto del edificio.

Defectos constructivos: Evidentemente que un defecto constructivo de la estructura resistente, además de disminuir la capacidad sismoresistente, puede ser la causa del escalonamiento de la misma. En efecto, puede dar lugar a un momento torsor imprevisto.

Cálculo estructural errado: Al igual que los defectos constructivos puede ser causa de disminución de la capacidad sismo-resistente y del escalonamiento imprevisto de la misma.

\section{REFUERZO DE LA CAPACIDAD SISMO-RESISTENTE DE EDIFICIOS CONSTRUIDOS}

Los últimos terremotos que afectaron ciudades construidas satisfaciendo normas sismo-resistentes vigentes a la fecha, evidenciaron un hecho que no podemos seguir desconociendo y es que muchos edificios actualmente considera- 
dos sismo-resistentes carecen de una adecuada compatibilización del diseño estructural con el diseño arquitectónico, pudiendo facilitar el escalonamiento de su sismo-resistencia y producirse el colapso parcial, y también total, por esta causa.

El desastre provocado en la ciudad de México por el terremoto que la afectó en septiembre de 1985 ha mostrado la importancia, hasta ahora desconocida de la arquitectura sismo-resistente, en el tema de la sismo-resistencia y del refuerzo de edificios como un efectivo aporte a la prevención de los efectos sísmicos.

En sintesis, frente a la necesidad de evaluar la capacidad de resistencia sísmica de un edificio construido y desde nuestro enfoque, se debe comenzar por detectar toda interferencia que proviniendo del diseño arquitectónico, conduzca a un escalonamiento de su capacidad resistente al sismo y proponer su solución a través de un equipo interdisciplinario para que la misma no afecte la funcionalidad de la obra arquitectónica.

Finalmente se pasará a considerar la conveniencia de reforzar la estructura sismo-resistente, propiamente dicha, de resultar necesario satisfacer un coeficiente sísmico mayor.

\section{PROPUESTA METODOLOGICA}

La eficiencia de la propuesta sismo-resistente, no es sólo función del sistema estructural, sino y fundamentalmente, de su compatibilización con el diseño arquitectónico.

En síntesis: La metodología propuesta se sustenta primero en un enfoque totalizador e integrador teniendo en cuenta que, en la respuesta al sismo de un edificio, es responsable no sólo la estructura resistente, sino todos los elementos que materializan el edificio y la ciudad, los aspectos espacio-formales, funcional, estéticos y económicos. En segundo lugar de la compatibilización del diseño arquitectónico con el diseño estructural, es decir, satisfacer el "principio fundacional» en todos los casos sin excepción y, además, satisfacer objetivos de eficiencia y optimización de su capacidad de resistencia sísmica desde el diseño arquitectónico.

A continuación se sintetizan los siguientes aspectos que condicionan los procesos de proyectación edilicia, a saber:

\section{A) Son variables referenciales:}

a) La naturaleza dinámica de la excitación sísmica.

b) El período predominante del terreno.

c) Epicentro cercano y lejano. d) Intensidad sísmica.

e) Sistemas estructurales.

f) Ductilidad.

g) Edificios flexibles o rígidos.

h) Sistemas constructivos.

B) Son requerimientos del diseño estructural sismo-resistente:

a) Sistema resistente tridimensional (comportamiento espacial).

b) Edificios livianos, en función de materiales y sistemas resistentes que eviten masas innecesarias.

c) Edificios de configuración simple, preferentemente simétricos en planta y altura.

d) Evitar excentricidades entre el centro de masa y el de rigidez.

e) Establecer los «grados de participación» de los distintos componentes edilicios en el fenómeno de la sismo-resistencia.

f) Relaciones rigidez-resistencia balanceadas entre los distintos elementos y/o subsistemas del mecanismo sismo-resistente, evitando incompatibilidades peligrosas.

\section{C) Son constantes compatibilizables:}

a) Comportamiento espacial de los edificios bajo cargas sísmicas.

b) Fuerzas sísmicas proporcionales al peso de las construcciones.

c) Cada elemento resistente absorbe una fuerza sísmica horizontal, independiente de su ubicación en planta, proporcional a su rigidez horizontal (caso de momento torsor cero). Esto permite disponer su ubicación de manera de poder absorber eficazmente los efectos de un momento torsor y también atender requerimientos del proyecto arquitectónico.

d) Las excentricidades de masa en planta y altura, generan torsiones inconvenientes.

e) Los mecanismos resistentes deben diseñarse de modo que todos sus elementos resistentes actúen simultáneamente.

f) Características de ductilidad e hiperestaticidad.

g) Rigidez y flexibilidad (interacción sueloestructura). 